\title{
Circuit
}

Musiques contemporaines

\section{Le mot, le son, le sens}

\section{The word, the sound, the meaning}

\section{Serge Provost}

Volume 3, numéro 2, 1992

Opéra? Gauvreau, Provost, Kagel

URI : https://id.erudit.org/iderudit/902052ar

DOI : https://doi.org/10.7202/902052ar

Aller au sommaire du numéro

Éditeur(s)

Les Presses de l'Université de Montréal

ISSN

1183-1693 (imprimé)

1488-9692 (numérique)

Découvrir la revue

Citer cet article

Provost, S. (1992). Le mot, le son, le sens. Circuit, 3(2), 21-44.

https://doi.org/10.7202/902052ar
Résumé de l'article

Le compositeur montréalais Serge Provost a mis en musique des extraits du livret d'opéra de Claude Gauvreau, Le vampire et la nymphomane, sous le titre L'adorable verrotière. Après avoir analysé les problèmes de la relation entre texte et musique chez Monteverdi et Boulez, Provost décrit l'aspect madrigalesque de son oeuvre et montre comment il l'a conçue à partir du texte de Gauvreau. d'utilisation que vous pouvez consulter en ligne.

https://apropos.erudit.org/fr/usagers/politique-dutilisation/ 


\section{Le mot, le son, le sens Serge Provost}

Je dédie amicalement cet article à tous ceux qui ont permis, par leur travail et leur dévouement, que le colloque et la création de l'Adorable verrotière ait lieu.

L'œuvre est dédiée à Madame Maryvonne Kendergi et aux merveilleux interprètes qui l'ont créée, le 3 septembre 1992, à Pauline Vaillancourt, à Lorraine Vaillancourt et aux musiciens du Nouvel Ensemble Moderne.

Écrire de la musique sur un texte et faire en plus œeuvre de théâtre représente pour le compositeur que je suis une sorte d'épreuve à traverser. Le texte se présente d'abord comme un obstacle me séparant du monde des sons que j'organise selon une logique de l'indicible.

La plongée dans l'univers du sonore provoque paradoxalement le silence, silence de la pensée discursive, abolition du mot, court-circuit. La conscience fonctionne alors sur un autre mode, selon un autre système de tension. Les images, les concepts transmutent directement en structures de sons (remplaçant les structures du langage), à la recherche de sens, de fonctions, le tout étant à organiser selon une dialectique du temps et de l'espace. Tout l'effort de la musique consiste à abolir le langage. "To quiet and sober the mind", avait dit un jour une musicienne indienne à John Cage.

aJ'ai voulu faire une pièce triste, et tout le monde a ri...! *

John Cage.

La musique a un sens qui ne veut rien dire.

Pourtant, depuis l'Antiquité, le langage est à l'origine de l'organisation du mısical, lui imposant d'abord les lois de la prosodie lorganisation du rythme et de la mesure, distribution des accents) puis les modèles syntaxiques (organisation fonctionnelle du mode en degrés prédominants et secondaires). Les formes de la musique occidentale naissent de la versification. La forme sonate éminemment narrative est le symbole de la musique-langage, une dissertation sonore. La révolution sérielle (en particulier l'œuvre de Webern) a fait éclater les structures discursives de la musique (et leurs fondements tonals). Le silence 
s'est introduit en toile de fond (une sorte d'aspiration vers le vide, dans l'esprit zen), purifiant ainsi le son de toute donnée sémantique - le son perçu en tant que matière s'adressant directement à l'esprit.

Ainsi le texte, revenons-y, m'apparaît comme un écran, une mer de mots où plonger - traversée des apparences - pour sonder les profondeurs du sens qui m'échappe. $\grave{A}$ travers des structures littéraires, retrouver le mouvement de la pensée; sous le poids signifiant des mots, retrouver l'image, la sensation. Trouver la pierre philosophale qui, du creuset, fera jaillir la musique.

\section{Relectures}

L'exercice consiste à interroger certaines œuvres du passé, proches ou lointaines, comme des oracles, afin qu'elles nous livrent le secret de l'alliance subtile de la poésie et de la musique ... et que, du tressaillement de cette union, émerge une forme de théâtralité.

Voici quelques réflexions à propos de:

MONTEVERDI - Madrigali guerrini e amorosi", "Hon ch'el ciel e la terra...*, livre VIII, 1638. * Hon ch'el ciel e la terra e'l vento tace e le fere e gli angelli il sonno offrena. Notte il carro stellato in giro mena e nel suo letto il mar seny'onda giace. »

Traduction: «Alors que le ciel et la terre et le vent se taisent, que le sommeil dompte les fauves et les oiseaux, la nuit promène son char étoilé et que dans son lit la mer, sans une onde, repose...

Premier tercet d'un sonnet de Pétrarque

Voilà un exemple remarquable où le compositeur s'attaque à un texte d'une qualité littéraire exceptionnelle. Un poème auquel on ne semblerait pouvoir rien ajouter, possédant sa propre musique sous-entendue. Pourtant ... la poésie; travail du rythme, de l'harmonie, de l'image, autorise la dérive du sens vers la métaphore. S'inscrivant dans cette mouvance, observons l'art du compositeur (cf. ex. 1).

La musique s'adapte à la prosodie, elle en élargit la respiration, en souligne délicatement les accents. Toutefois, l'organisation matérielle des sons (un accord de tonique mineure en position de quinte, dans le grave des voix, accord initial prolongé par un silencel installe déjà tout un espace, met en 
Exemple 1

Hor ch'el Ciel e la Terra
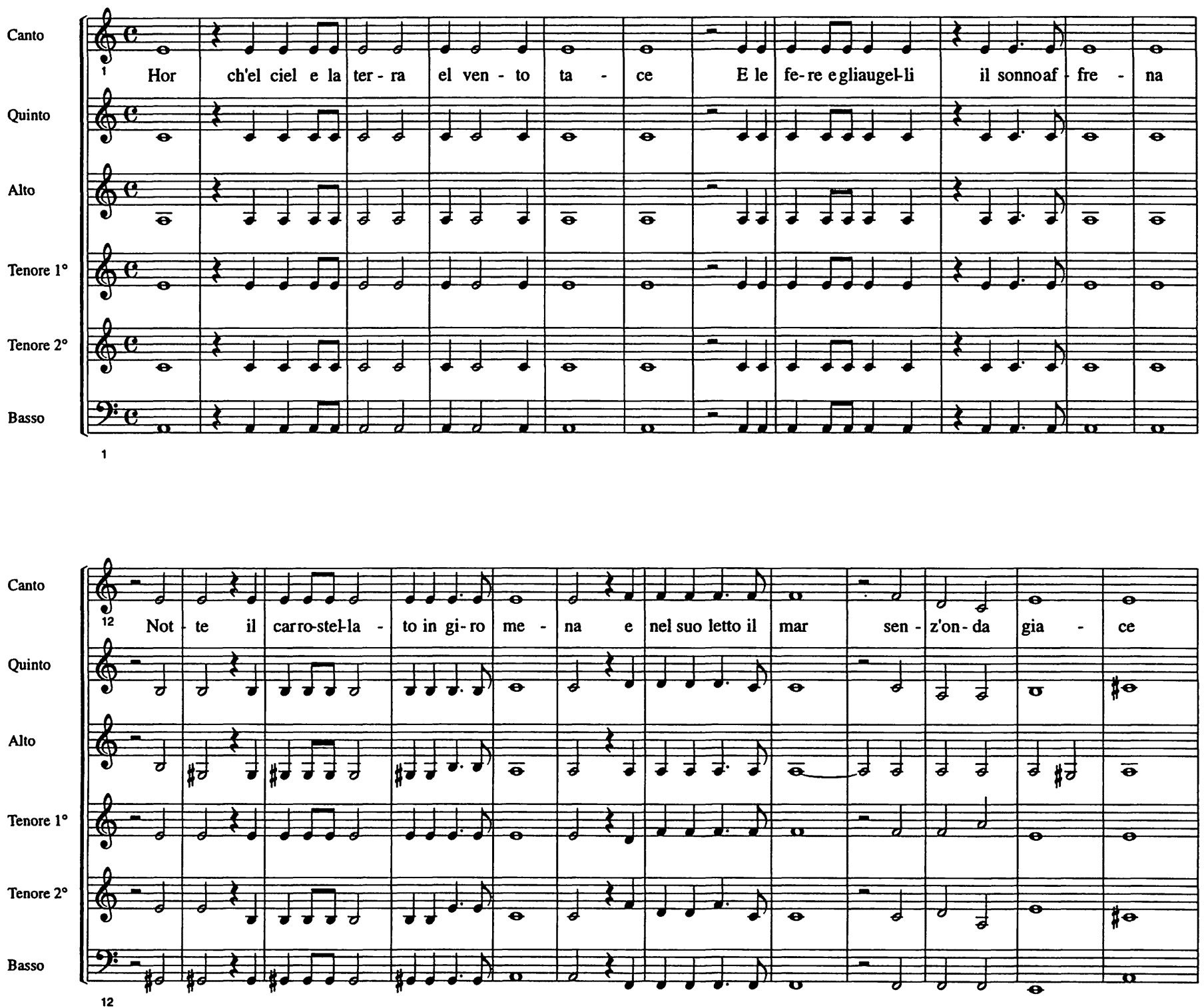
scène le premier mot Hon. C'est déjà tout l'univers nocturne ainsi suggéré dans son expectative. Tout le reste du vers et le vers suivant sont déclinés dans la projection de cet espace ouvert par le premier accord, amplifié par les silences - respiration profonde du temps correspondant à l'espace.

Notte, introduisant le troisième vers, la nuit est nommée par l'apparition événementielle de l'accord de dominante et promu au rang de personnage. Enfin, au quatrième vers, l'élargissement de l'harmonie et la résolution du retard à la dernière mesure suggèrent l'immensité calme de la mer - tout attend...

Par des moyens très simples, nous assistons à la naissance d'un théâtre imaginaire où se fondent le mot et le son pour l'avènement du sens dans toute son amplitude. Véritable alchimie: le mot devenu musique et la musique, poésie, investie de tout son pouvoir de représentation. Cette investigation de l'univers métaphorique dans le domaine musical révèle Monteverdi dans toute sa modernité, dans toute son actualité.

Cette alchimie monteverdienne trouve son accomplissement dans Pli selon pli de Boulez. Cette collection de cinq pièces (Don, Improvisations H-H-III sur Mallarmé et Tombeau) sur des poèmes de Mallarmé (Don du poème/ Le vierge, le vivace, et le bel aujourd'hui/Une dentelle s'abolit/ $\grave{A}$ la nue accablante tu/Tombeaul constitue une réflexion approfondie sur l'univers poétique.

Jeux de miroirs, double portrait (du poète et du compositeur): ici le phénomène de la transmutation est poussé à d'ultimes limites. Dans la première pièce, Don, la musique est littérallement enceinte du verbe, dont elle annonce symboliquement la naissance en correspondance avec le seul vers: "Je t'apporte l'enfant d'une nuit d'idumée!». Par ailleurs, des "réminiscences préalables" (Boulez), fragments de poèmes à venir dans le reste du cycle, évoquent une sorte d'accouchement. Par contre, dès la seconde pièce, la voix, au premier plan, incarne le texte dans un style nettement madrigalesque. Comme chez Monteverdi, la forme générale est issue du sonnet; la ligne du chant, assez ornée, épouse en ses figures les contours et l'imagerie du poème. L'effectif instrumental se voit cantonné dans le commentaire harmonique, résonances, extrapolations timbrales de la ligne principale. Toutefois, dans Une dentelle s'abolit, le transfert du littéraire vers le sonore opère à nouveau, le commentaire instrumental en est chargé. Dans les deux autres pièces, le texte se résorbe et meurt, ainsi la musique fécondée prend tout l'espace. 
Cette œuvre pose de façon exemplaire le problème de la relation au texte. Peut-on impunément le manipuler, l'amputer, le disséquer sans en trahir le sens? L'intelligibilité directe du mot, de tous les mots, estelle nécessaire à l'entendement? l'œuvre musicale en tant que réponse à ces questions propose la perception du poétique en tant qu'expérience sensorielle, selon un système dépassant la correspondance baudelairienne; il s'agit d'une volonté de réincarnation du verbe par la musique. Si le projet touche son but, le compositeur a-til trahi le poète? Luigi Nono adoptera une démarche similaire, colorée toutefois par la teneur politique de son propos. Les textes révolutionnaires employés dans Comme un fleuve de force et de clarté donnent naissance à une musique singulièrement puissante, survoltée par le message qu'elle contient.

\section{Le mot en tant qu'objet}

Les œuvres que l'on vient d'aborder sont conditionnées par un processus symbiotique, où la distance entre le mot et le son musical tend à s'abolir. Toutefois, certaines œuvres nous montrent une relation tout aussi féconde, fondée sur la distanciation et l'effet poétique qui en résulte.

Dans les cycles de Lieder de Schubert et de Schumann, les poèmes sont portés par des mélodies de factures généralement assez simples, presque anodines, et qui ne mettent aucune entrave à la perception lla plus naturelle possible) du texte. Voilà que le sens, le drame du poème se réfugie dans l'accompagnement, de facture plus élaborée, contenant maints éléments figuratifs ou symboliques. Le piano met en scène le poème. Le mot non résorbé devient objet sonore, abdiquant une partie de ses fonctions au profit de la musique. Dans le Pierrot lunaire de Schœnberg, par le procédé du Sprechgesang, le motobjet se distancie davantage par la perte de définition mélodique qui le reliait à l'harmonie. Le mot information gagne en autonomie, se détache, tandis que la musique instrumentale extrapole l'univers poétique qui l'environne. Cette confrontation des véhicules du sens modifie la perception; une tension s'installe entre le mot et le son, créant un nouvel élément de théâtralisation. La présence de récitants (textes déclamés) dans certaines œuvres de Marius Constant ou de Maurice Ohana et, plus près de nous, chez Claude Vivier (Prologue pour un Marco Polo par exemple) accentue cette tension du texte et de la musique. Ainsi un territoire de possibilités 
expressives s'installe entre le chant et la déclamation. La notion de théâtralité investit ce territoire et devient un paramètre supplémentaire s'immisçant entre le littéraire et le musical.

\section{Hörspiel}

La période de l'entre-deux-guerres a vu l'apparition d'un genre spécifique, la dramatique radio. Un art de l'évocation, art aveugle s'adressant directement à l'imaginaire par le canal privilégié de l'ouie. La musique en général y fait figure de faire-valoir (du moins à l'origine), mais l'art de la prise de son, l'évocation de lieux, d'atmosphères, le traitement des voix, l'art du bruitage ajoutent une nouvelle panoplie de moyens expressifs. Cet art en trompel'oreille utilise des artifices (conventions propres au théâtre) créant de nouveaux archétypes de la perception. Par exemple, une simple réverbération suffit à évoquer les voix du passé ou celles du rêve. Cet art radiophonique appartenant lui-même au passé fait figure de souvenir sonore et possède une perspective spatio-temporelle dont l'effet de distance /comparable au film en noir et blanc) peut être particulièrement expressif. Sa reconstitution par des moyens électroacoustiques, à incorporer dans une œuvre musicale, peut offrir des solutions intéressantes.

Ces quelques réflexions mettent en perspective les questions qui m'ont préoccupé préalablement à mon travail de composition.

\section{Le théâtre ef son ombre}

L'adorable verrotière est un mélodrame que j'ai composé à partir d'extraits du Vampire et la Nymphomane de Claude Gauvreau, livret d'opéra écrit en 1949 et destiné au compositeur Pierre Mercure. Ce dernier n'en fit toutefois jamais la musique. II faut dire que le texte de Gauvreau n'a absolument rien du livret d'opéra conventionnel, c'est-à-dire d'une sorte de pièce théâtrale adaptée, dont les dialogues, assez simples et volontiers descriptifs, doivent se conjuguer aisément à la musique. 
Tout au contraire, la pièce de Gauvreau est une poésie au style impérieux et d'une extraordinaire violence, dont le registre d'expression va de la souffrance vive à l'acharnement et à la révolte, et atteint parfois des moments d'illuminations sublimes. II n'y a point d'action telle, sinon en tant qu'imaginaire. Pourtant les personnages sont de véritables héros d'opéra dans l'accomplissement tragique de leur destinée. Le langage, d'une richesse inouie, foisonne d'images, telles des hallucinations. Le ton est épique: ce qui soustend tout ce texte, c'est un formidable geste de libération.

Le texte du Vampire est monumental, il se poursuit avec une densité soutenue sur une trentaine de pages. La mise en musique de la pièce dans son intégralité appelle l'opéra ... et la mise en œuvre de vastes (et onéreux) moyens. La Société Royale du Canada m'avait commandé une œuvre avec voix d'une durée de vingt minutes. Que faire? Ce texte me hante et m'attire irrésistiblement! Toute la question est de trouver le moyen d'aborder l'œuvre dans une version abrégée sans en trahir le contenu.

Après avoir traversé (après maintes relectures) la trame extrêmement dense de cette langue exploréenne propre à Gauvreau me sont apparus les piliers d'une architecture dramatique assez proche de la tragédie classique: d'abord une action préalable en forme de catastrophe. La naissance du vampire, puis l'exposition des principaux personnages, le vampire lenfant ef adultel, la nymphomane, enfin le dénovement tragique, la mort des personnages héroïques. Des points d'ancrage me sont apparus de plus en plus clairement, me permettant d'extraire les portions de textes nécessaires à la constitution d'un mélodrame conservant la logique essentielle du texte intégral. Par ailleurs, Gauvreau (dans les notes accompagnant la pièce) suggère trois phases:

a) La naissance du vampire

b) L'amour

c) La nymphomane

En extrapolant ces données, je suis arrivé à une construction en cinq tableaux: Prélude, la naissance, le vampire, la nymphomane ef l'aliéniste, la mort des héros.

- L'adorable verrotière sicolait sur les mitres de porcelaine.

L'adorable verrotière sicolait sur les haies blanches de chocolat.

Et la poudre pas caline sicolait dans la blafarde soleillimure le temps sué des albâtres »

(Claude Gauvreau) 
Ces premiers vers inaugurent le texte. Cette adorable verrotière est un personnage mystérieux, elle préside à la naissance du vampire et, en fait, de l'œuvre même. Personnage secondaire, elle représente une forme d'antériorité (d'outre-tombe) qui plane sur tout le drame. C'est pourquoi je l'ai choisie pour figurer dans le titre du mélodrame.

J'ai utilisé une voix de soprano (chanteuse-récitante) et un ensemble instrumental, composé d'une flûte, d'un violon, d'une clarinette basse, d'une contrebasse, de percussions, ensemble auquel s'ajoute une bande magnétique.

La percussion représente le principe actif - incidences formelles prédominantes, rôle polyvalent. Elle découpe la forme, préside aux événements dramatiques (utilisation du tam-tam). Elle provoque, met en branle, interrompt brutalement (l'enclume emblématique de l'inéluctable), met en relief les éléments structuraux. Fait figure de symbole (un carillon de verre ébranlé par un ventilateur emprisonne l'âme de l'adorable verrotièrel, la machine à vent (héliophone): idée du souffle, balayage, volatilité du temps, évocation des machines de théâtre, le tambour militaire, etc. En fait, la percussion incarne le rôle de la tragédie.

Bien que les autres instruments soient utilisés sur un mode moins théâtral, ils ne sont pas dépourvus d'éléments de représentation. La contrebasse, de par sa nature, assure le fondement de l'édifice sonore et l'utilisation de nombreux sons harmoniques (diffraction des sons fondamentaux) établit le spectre général, définissant ainsi le cadre spatial d'où les autres éléments (harmoniques, mélodiques) pourront surgir. Les instruments à vent, utilisés surtout dans le troisième tableau "Le vampire», sont volubiles, virtuoses, d'une écriture très variée; à la fois contrepoint serré de la voix et commentaires du texte, ils incarnent la fugacité du personnage. Le violon, quant à lui, se détache par moments en un contre-chant au caractère lyrique, et, par ailleurs, il participe à l'action générale.

La bande magnétique (de style typiquement radiophonique) a pour fonction d'établir un effet de perspective spatiale et temporelle, Hörspiel intégré au mélodrame. Les interventions de cette bande souvent en "coup de théâtre " ont une fonction hautement dramatique et une incidence formelle évidente, assurant la cohésion entre les tableaux. La diffusion d'éléments pré-enregistrés crée forcément un "ailleurs" dans le temps de par son traitement: fonction d'antériorité sur laquelle on reviendra.

La voix est évidemment l'élément central, la cheville ouvrière de l'œuvre. Le texte de Gauvreau, possédant un registre expressif très étendu, oblige à dépasser les limites du chant. Le plus large éventail des possibilités vocales est ici requis. 
Afin de mettre en lumière la description des éléments sus-mentionnés, je propose d'en examiner la mise en œuvre (en action) dans chaque tableau, à l'aide d'exemples musicaux tirés de la partition.

\section{Prélude}

Ce prélude est une ouverture, un lever de rideau. Trois coups d'enclume (réminiscence du théâtre) nous projettent déjà dans l'action dramatique initiale : la naissance de la musique (premier personnage, déesse de l'espace ef du temps) par l'incarnation du son, la partition en étant la mise en scène. l'enclume provoque, l'héliophone féconde. Le vent insuffle l'anima aux bruits (grincements de l'arco-grain) et le transforme en être sonore, de même, par l'action du souffle, les carillons de verre /réminiscences préalables de l'adorable verrotière) s'animent. En sept mesures, l'espace harmonique (le lien du drame) est établi. À la huitième mesure, le silence (l'âme de la musique) cautionne cet espace et en informe la temporalité. Dans la projection de ce silence, la deuxième séquence, agrandissant l'espace sonore, préside à la naissance de la voix (préparée par le mouvement mélodique de la clarinette basse), émergence de la mélodie (intervalles générateurs du mode) et réminiscence anticipée de l'héroïne (plainte). En quelques mesures, tout l' « opéra" est déjà là en essence (sans les fausses colonnades et les lourds drapés!) (cf. ex. 2).

Par la suite, le prélude croît en intensité et se conclut par la première intervention de la bande magnétique (un véritable extrait d'opéra): c'est Norma se jetant dans le bûcher expiatoire! Une apparition fantomatique! le premier geste tragique est accompli, le drame peut avoir lieu. Place aux héros!

Mais ... tout est peut-être déjà consommé, l'œuvre à faire n'en est peut-être que la mémoire vive? Ambivalence de la perception du temps. 
Exemple 2

\section{L'ADORABLE VERROTIERE}

- prélude -

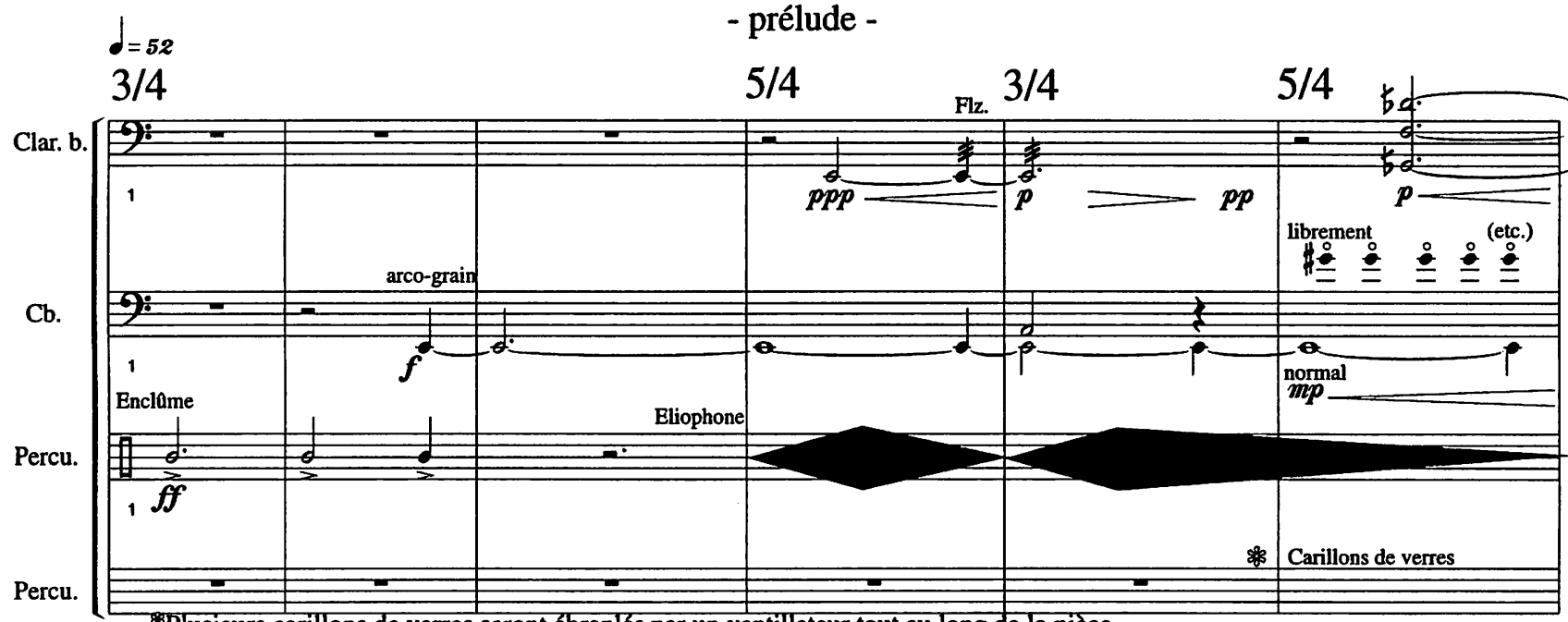

1 Plusieurs carillons de verres seront ébranlés par un ventillateur tout au long de la piéce.

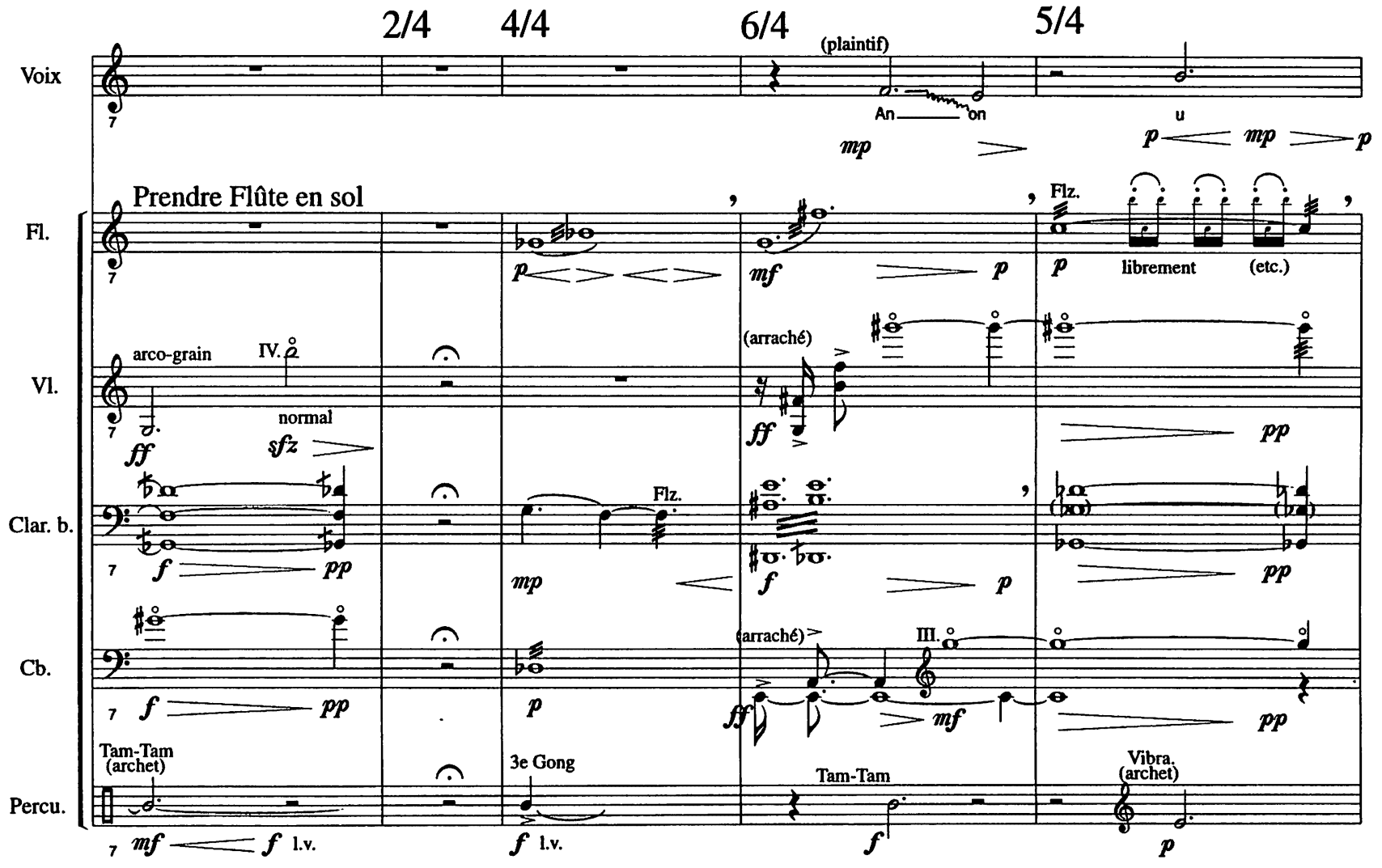




\section{La naissance}

L'action se situe dans le passé, un narrateur, \&l'homme aux deux pieds bots , nous relate les faits...

«L'adorable verrotière sicolait sur les mitres de porcelaine."

Une autre voix est nécessaire, un autre temps, un autre lieu. Évocation du poète disparu lisant son texte à la radio, par exemple; référence à l'époque où Gauvreau écrivait « Le vampire...», 1949. Plus loin dans le temps, l'image de Lautréamont hurlant à la nuit les "Chants de Maldoror» en tapant sur son piano. Ici la bande magnétique trouve un emploi privilégié, la reconstitution suggérée d'un vieux Hörspiel: la voix du récitant mixée à un piano lointain (réverbéré) et à un bruitage suggérant la pluie. Pour créer l'effet de perspective, la bande se superpose en filigrane sur l'ensemble instrumental, qui poursuit son cheminement de façon quasi indépendante. La voix sur scène, sur un texte commentaire (la mère du vampire), se voit confier des mélodies très simples et des éléments de Sprechgesang proches du murmure. Elle est dans l'expectative (cf. ex. 3).

À la fin de ce tableau, les deux éléments sonores se rejoignent en intensité, la chanteuse émet un si aigu lécho de Normal, puis au son du tambour militaire, la catastrophe est annoncée.

« L'enfant au foie de tourmaline, aux viscères de carlotes, est né. *

Silence - sur bande: cri de mort de LULU, voix d'outre-tombe, autre héroïne sacrifiée, anticipation de la "nymphomane».

\section{Le vampire}

Ce tableau contraste fortement en regard du précédent, et son traitement en est fort complexe. Il s'agit de l'exposition du premier personnage principal, le vampire. Le texte porte la mention: «le vampire enfant». II s'agit d'un texte d'une exubérante vitalité où les images foisonnent à toutes les phrases. Les personnages incarnent toutes les forces à la fois créatrices et destructrices de la jeunesse. Le ton est tout ensemble enjoué, sensuel, emporté, violent. II ne 
Exemple 3

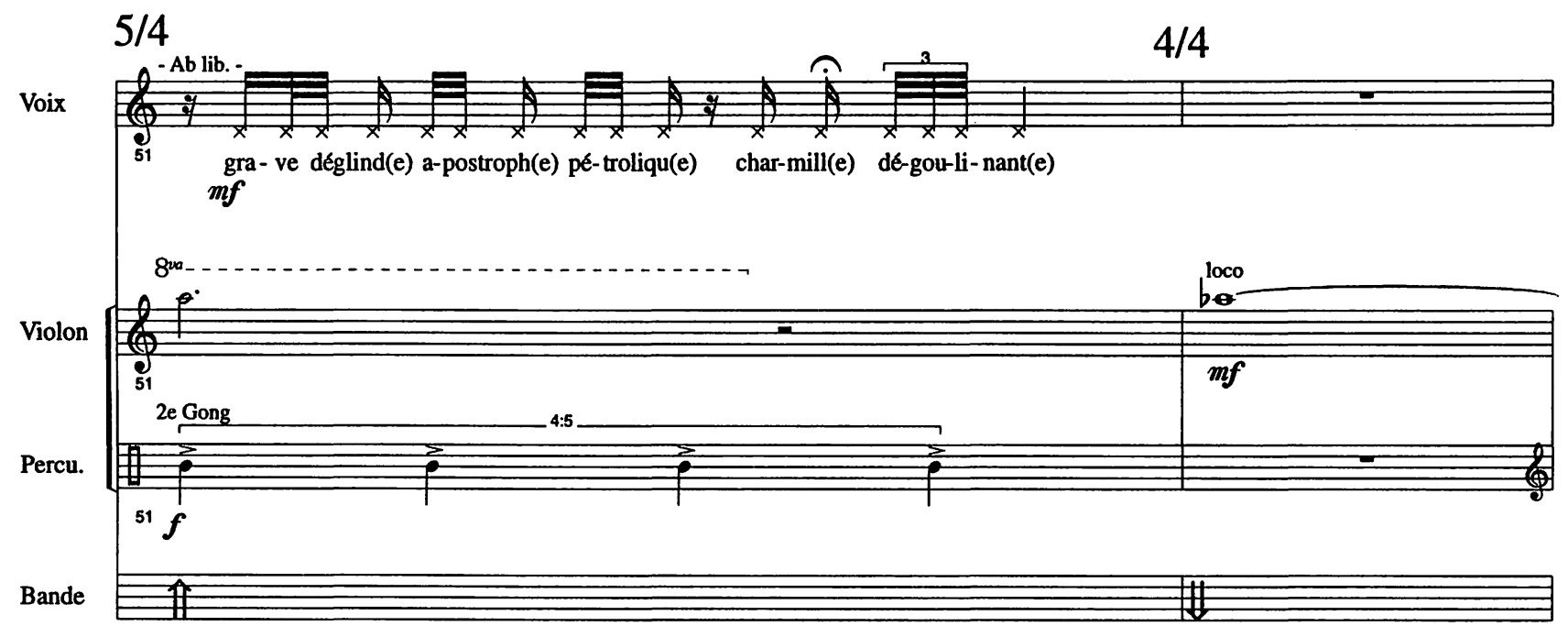

51

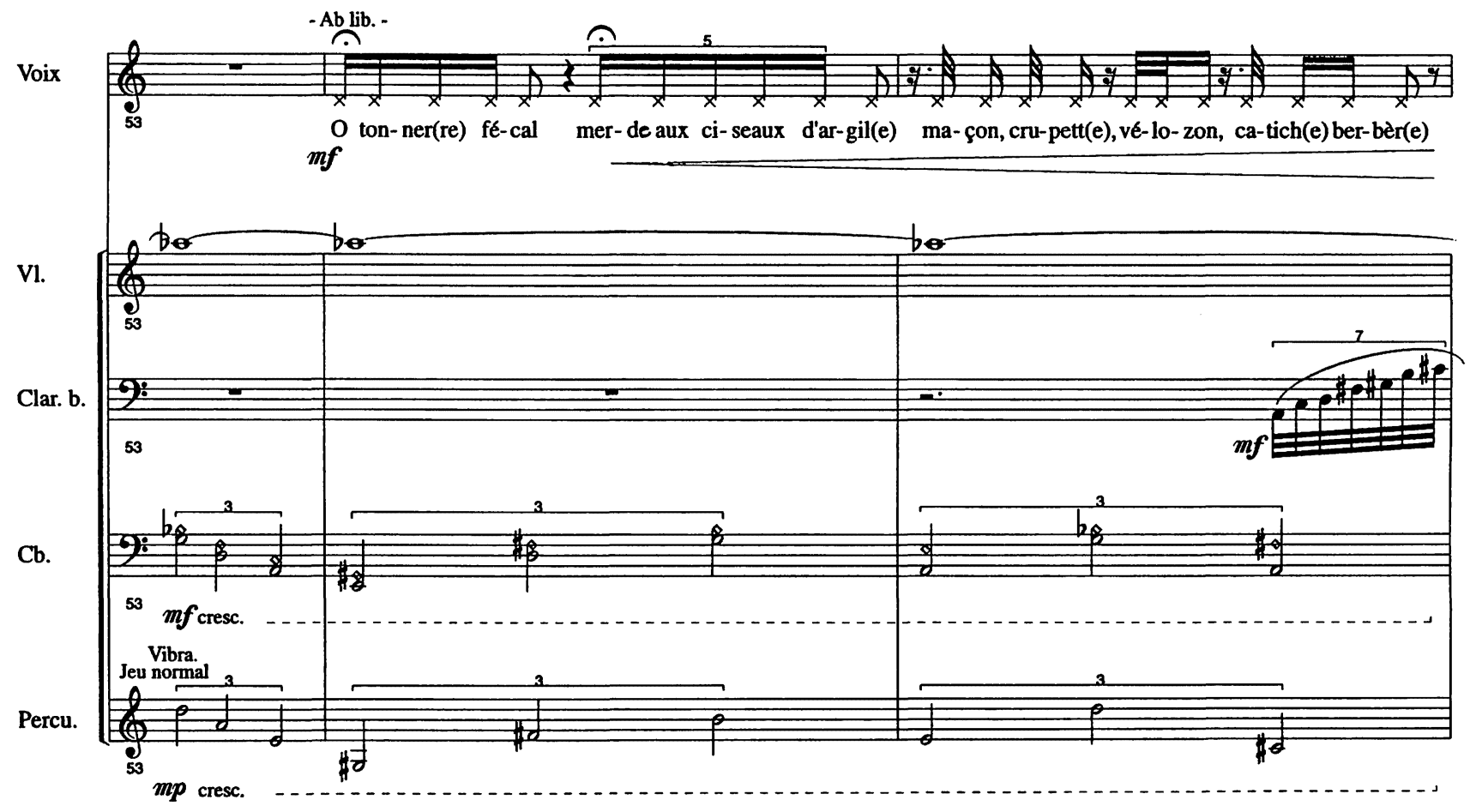


s'agit plus d'un «passé antérieur» où planait l'ombre de la tragédie, mais d'un présent qui se consume. Si la "naissance" a reçu un traitement en forme de Hörspiel, le "vampire" sera traité à la façon d'un madrigal. Donc, aucune intervention de bande magnétique pendant ce tableau.

Le madrigal requiert sept sections pour s'adapter au découpage du texte. l'écriture vocale et instrumentale sera nerveuse, détaillée et d'une grande fugacité. La voix chantée sera privilégiée, entrecoupée de sections en Sprechgesang (plus près du chanté que du parlél. Gauvreau, dans ses notes au compositeur, suggère pour le vampire une voix de basse, mais pour ce texte, il faut une voix agile au timbre clair, un soprano convient davantage. (cf. ex. 4)

Les premiers mots, forts juvéniles, s'expriment aisément par un Sprechgesang exclamatif qui lance le contrepoint échevelé des instruments (section I du madrigal). Par contre, le texte devient plus lyrique et le chant, assez orné, proche de l'esthétique baroque, trouve sa place. (cf. ex. 5)

«Où peinturer en blanc les trajectoires du pommier fou de véga?

$\mathrm{Ce}$ vers particulièrement somptueux est mis en valeur par une vocalise-récitatif, la voix émerge des instruments et le chant triomphe. (cf. ex. 6)

Par contre, le style haletant de la section suivante convient au Sprechgesang qui se communique aux instruments (pizzicati d'embouchure, pizzicati à la contrebasse). Vers le sommet de la ligne mélodique, le chanté prend le dessus sur le parlé. À noter: le violon qui se distingue du contrepoint canonique sériel, pour faire un contre-chant sur un mode en quart de ton. (cf. ex. 7)

* Parade crue, parade entamée, parade de papier maché que la rigidité des bonbons jaunissants transforme en hermitage d'acier brut. "

Fidèle au stile rappresentativo de Monteverdi, cette section est une pure image sonore. Intervalle emblématique à la voix, septième majeure, marche militaire frénétique, violon endiablé; référence à Berg (Wozzeck), Zimmermann (Die Soldaten), Stravinsky (L'histoire du soldat). (cf. ex. 8) 
Exemple 4
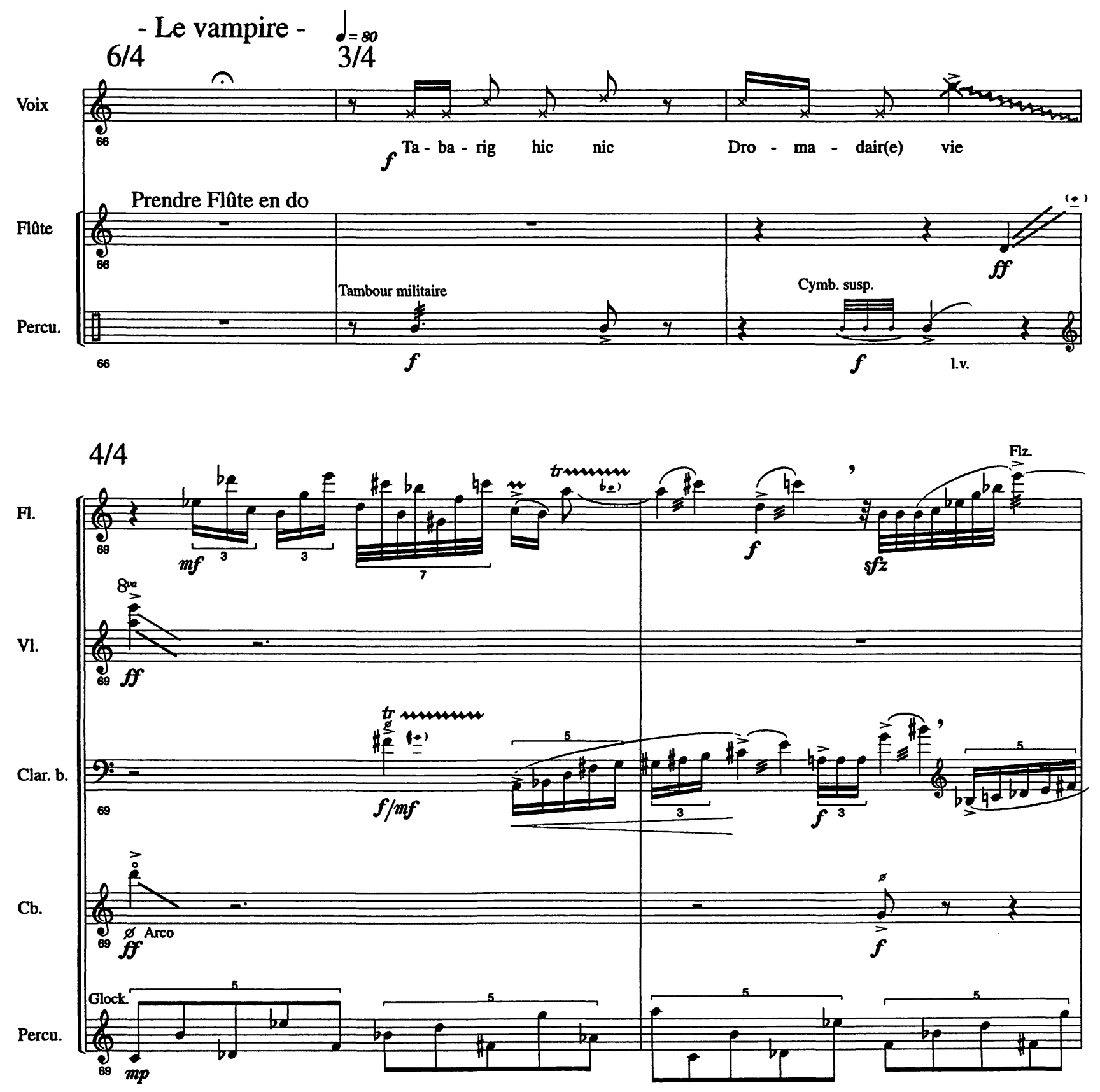
Exemple 5

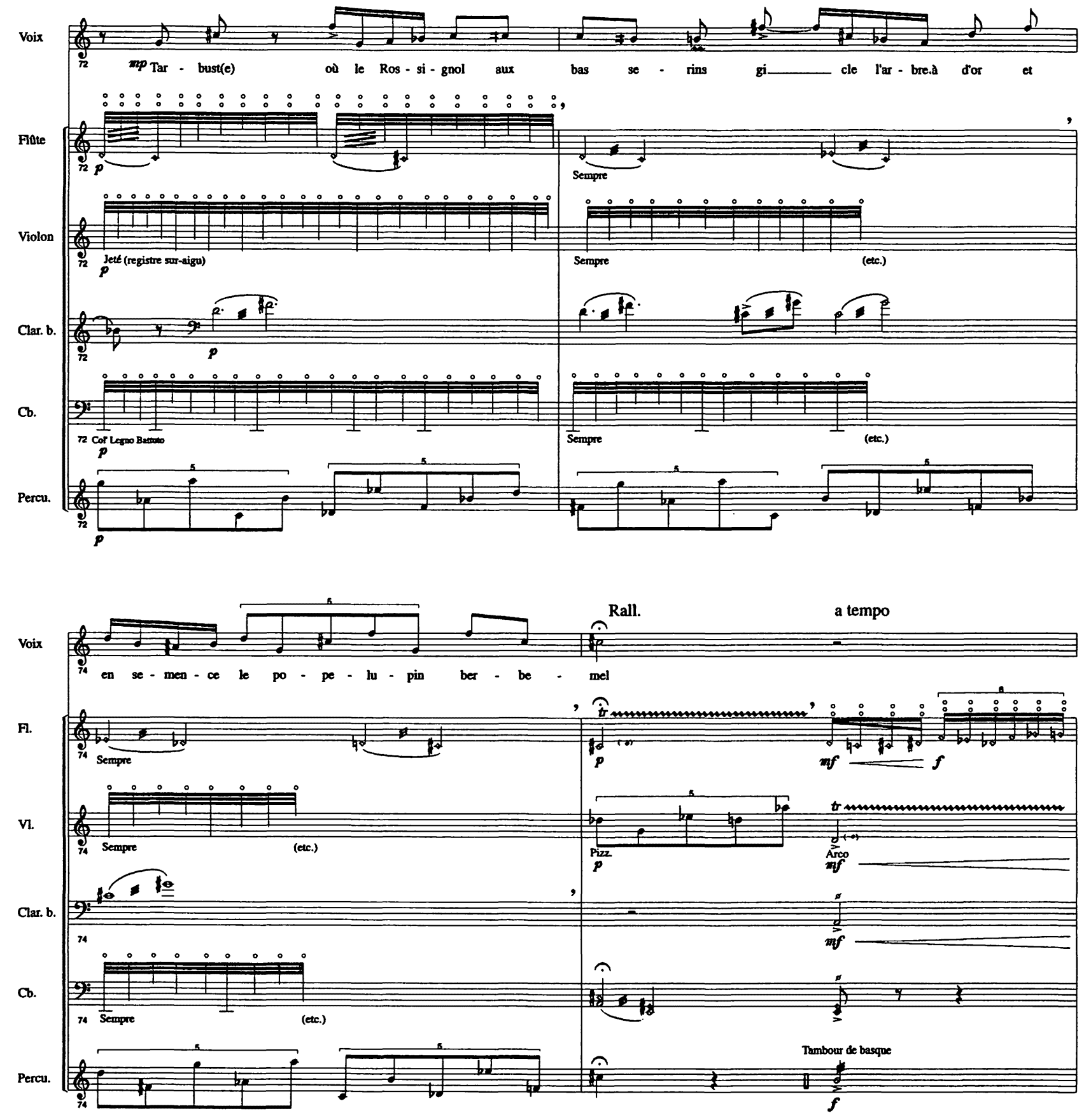


36

Exemple 6

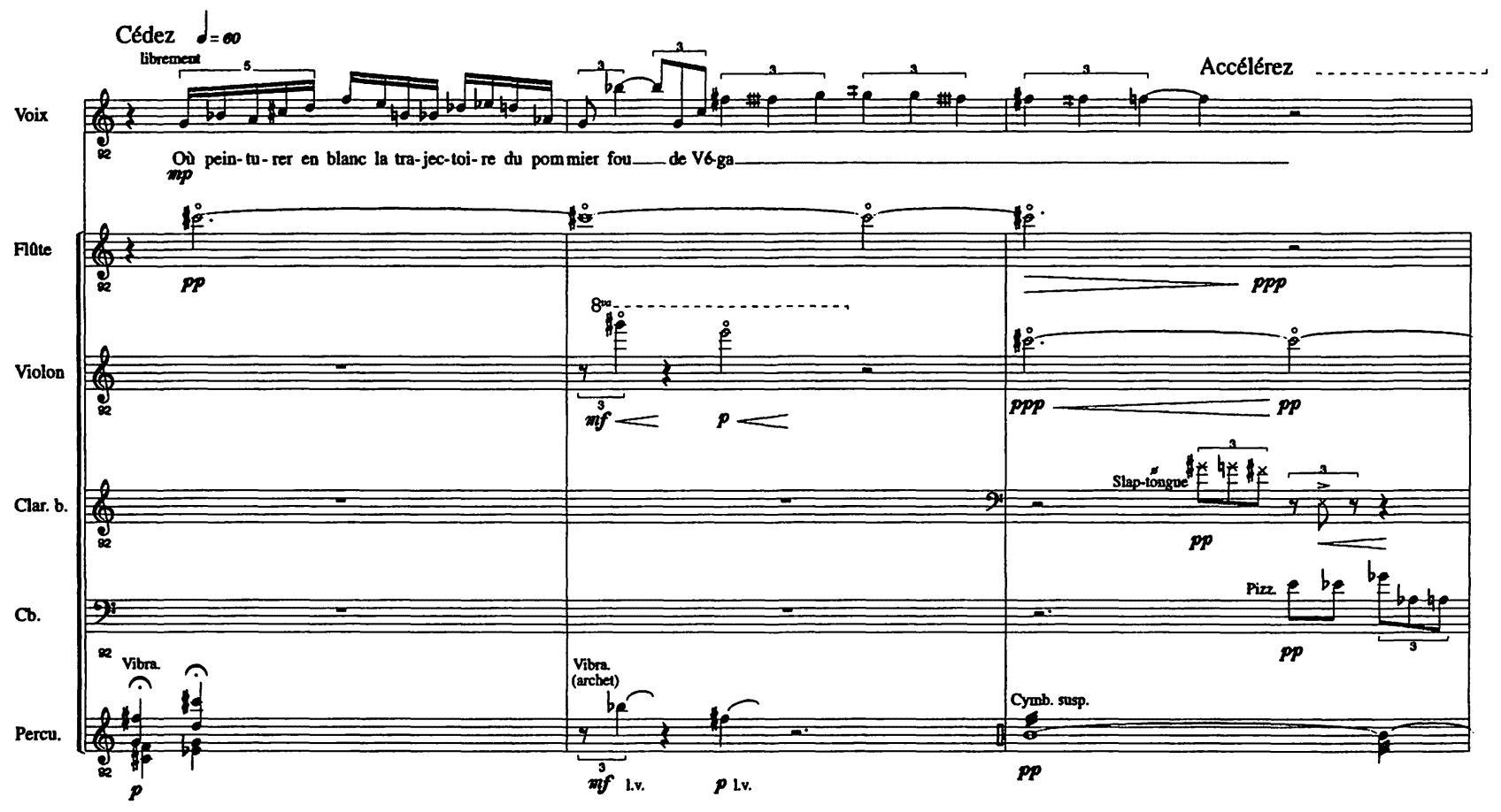


Exemple 7

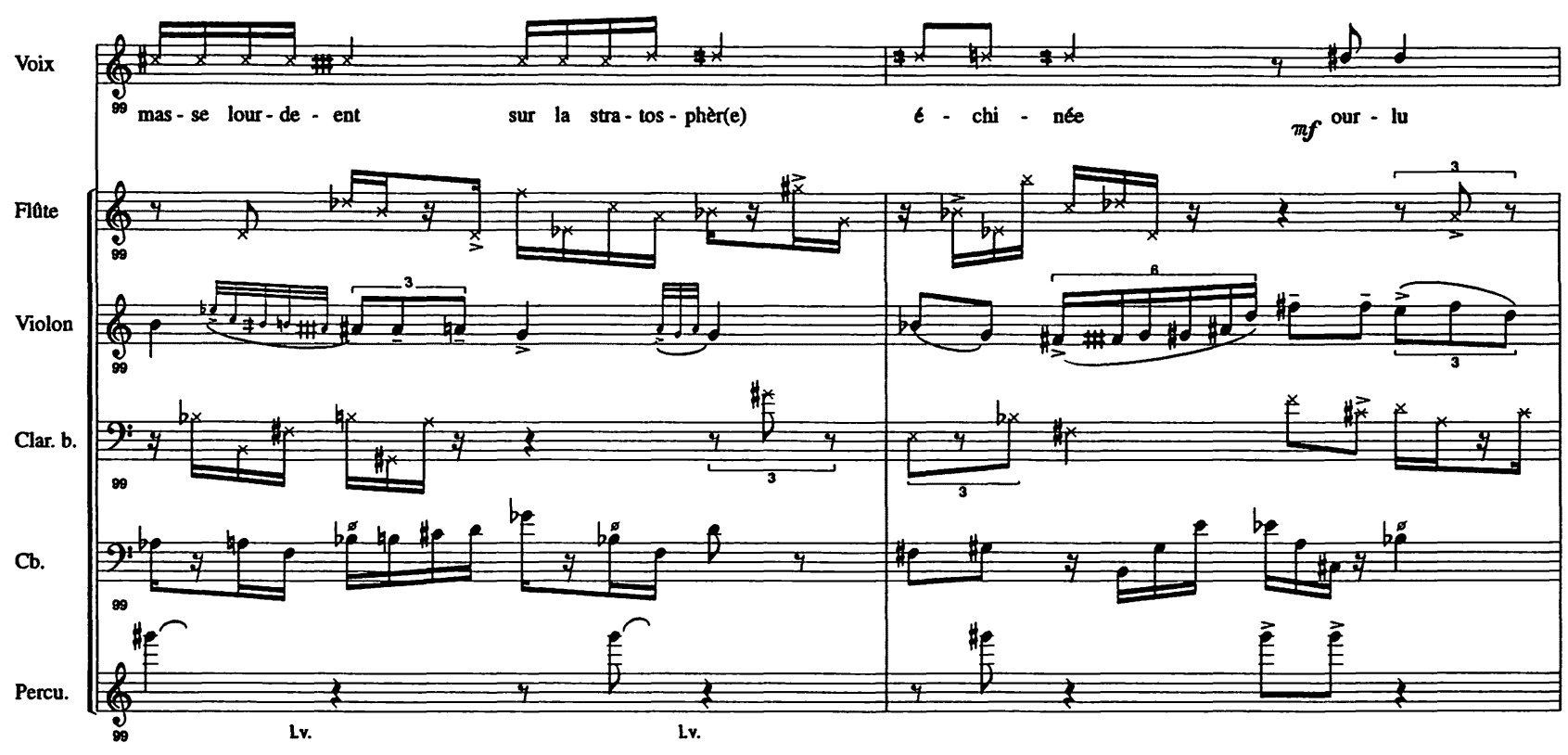

Voix

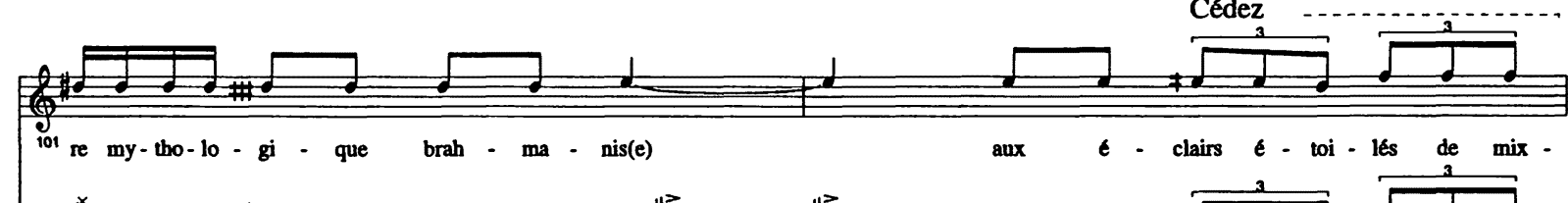

F1.

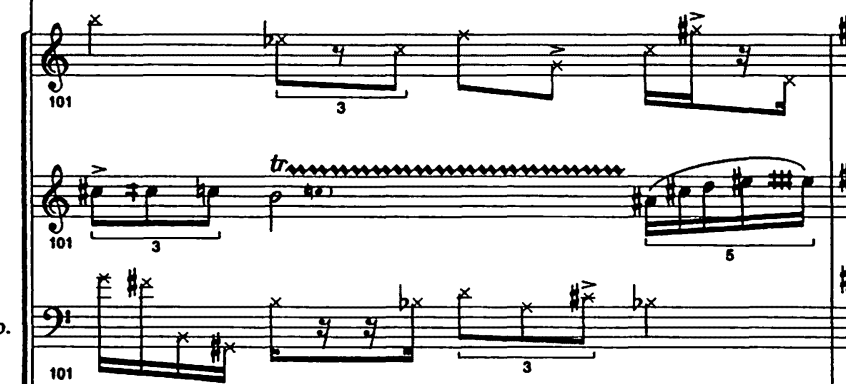

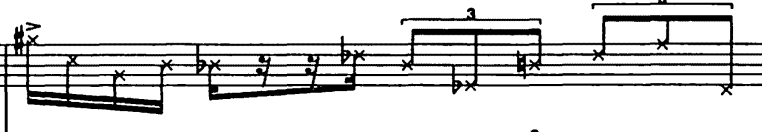

v1.

Clar.

Cb.
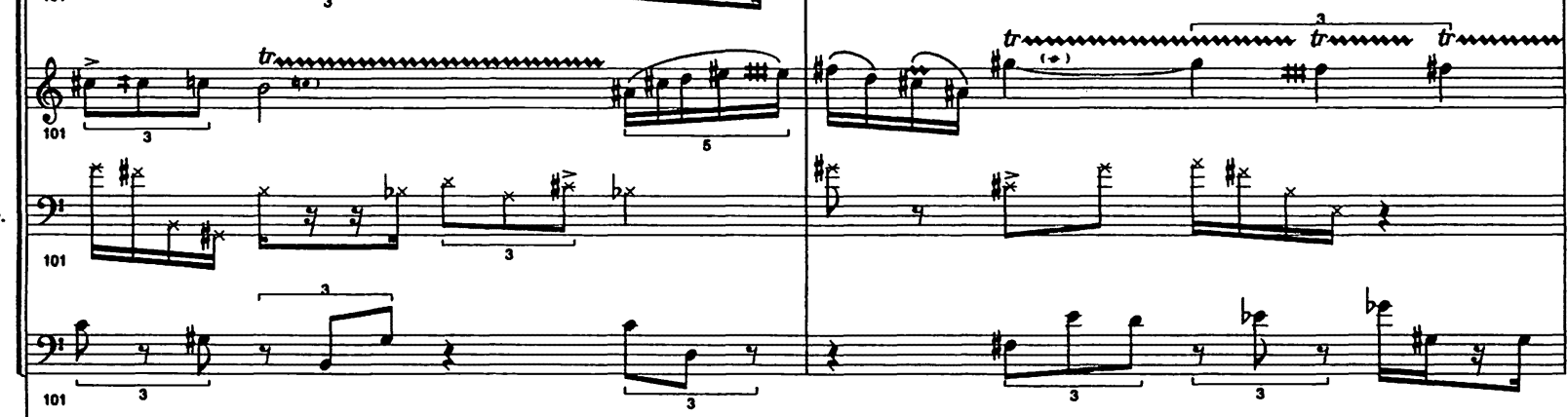

脂

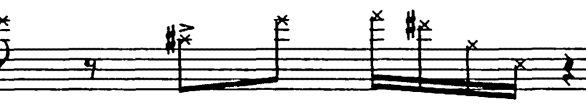

Percu.

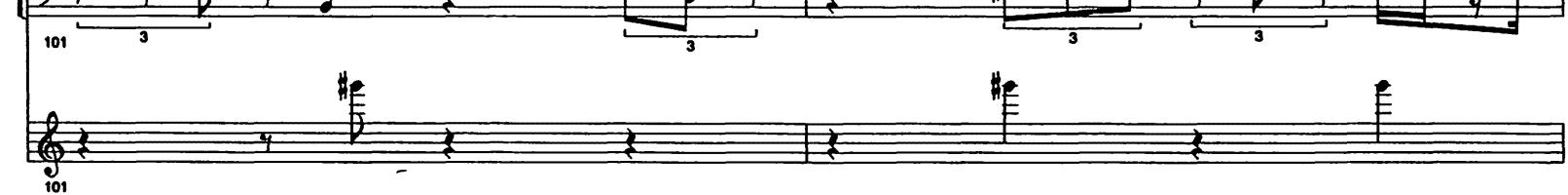


Exemple 8
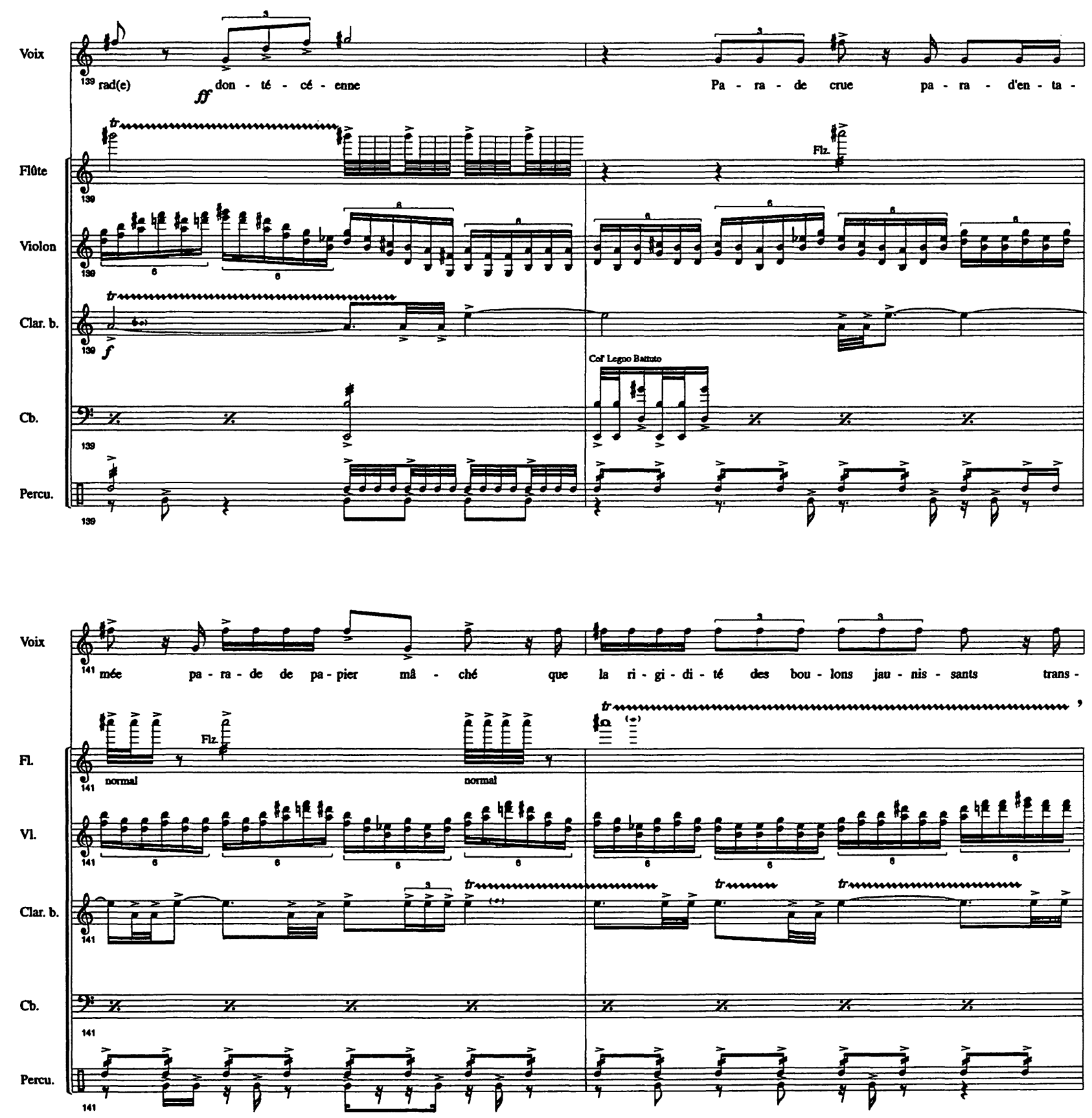


\section{La nymphomane}

Après une intervention de la bande magnétique: «Qui est cette femelle au cul de Paraguay".

La nymphomane paraît. Ce tableau est très particulier et nécessite un traitement tout à fait spécial. Le texte en forme de tirade, dans la plus pure tradition de la tragédie, atteint un paroxysme de violence à travers toute l'œuvre. Le contenu en est particulièrement poignant, références autobiographiques: scène dans un asile d'aliénés. Ce texte, publié ci-après en encadré, ne peut pas être chanté. Sa seule vérité se trouve dans la déclamation, la chanteuse doit se transformer en tragédienne. Toutefois, c'est là que la percussion prend toute son importance. La percussion devient un personnage à part entière, la tragédie incarnée. Elle extrapole toute la violence contenue dans le texte et devient un protagoniste de la récitante. (cf. ex. 9)

Au paroxisme de ce tableau, le percussioniste intervient en paroles et scande son texte de coups d'enclumes; il joue le rôle de "l'aliéniste". Parlant dans un mégaphone, sa voix devient métallique, à cela s'ajoute le bruit des sirènes, et, sur bande, le dialogue ultime de "Norma» et de son père la condamnant. Tous les éléments du théâtre tragique sont ici rassemblés.

Silence - bande: énorme éclat de verre.

«Ha! nordique écroulement."

\section{La mort des héros}

Dernier tableau: la destinée tragique s'accomplit. Les héros, couple uni par l'amour éternel, mais détruit par les forces obscures du mal, disparaissent et s'enfonçent dans le royaume des ombres. Figures du poète et de sa bien-aimée détruits par les démons de la médiocrité, de l'incompréhension et de la bêtise.

Toutefois, une tendresse immense se dégage de ce texte (dernière page du Vampirel; le traitement sonore n'a pas à être épique. Ici les instruments se taisent presque tout à fait. Ce dernier tableau renove avec le Hörspiel, 
Exemple 9

Voix

$4 / 4$

$2 / 4$

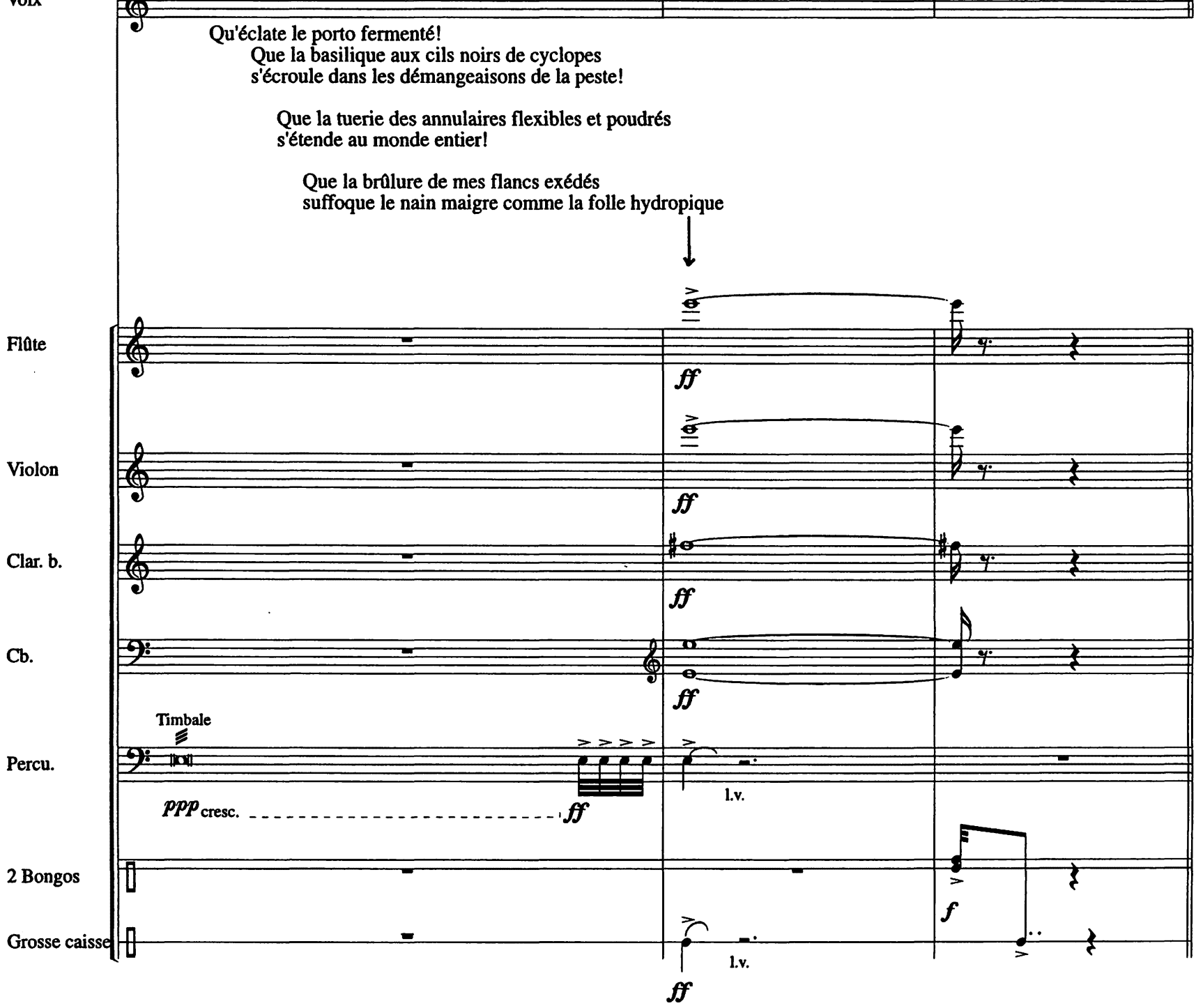


dialogue (duo) entre la voix sur bande et la chanteuse-récitante; celle-ci ne chante plus (sauf un ultime si aigu, écho lointain), elle se livre tout doucement au sommeil de la mort. La bande est peuplée de chants d'oiseaux sur une trame harmonique, lointaine résonance de toute la musique précédente. Tous les instrumentistes actionnent avec la chanteuse de petites boîtes à musique en signe d'adieu. Puis l'héliophone (machine à vent) qui avait donné vie à la musique (prélude) en emporte les derniers échos dans la mémoire et le temps, seule l'âme de l'adorable verrotière agite encore son petit carillon de verre.

*Ainsi si le pied nous foule, ainsi la gueuse nous tord, mais l'essence de chardons d'adieux, l'essence des exaltères épiques, ils ne la touchent pas. "

Claude Gauvreau 


\section{LE VAMPIRE ET LA NYMPHOMANE (extrait)}

\section{de Claude Gaurreau}

\section{LA NYMPHOMANE : Hou Hou Lou Lou Ou Lou Aou}

Ce fer rouge est un démon aux escalopes de cendre!

Le diamant putride me meurtrit, j'ai soif, je tombe dans les neiges de charbon rouge!

Ce tortionnaire aux breloques de printemps m'assouplit dans la tranchure des nerfs!

Je suis pendue par le nerf de mes aines à une potence de terre cuite où les rats et les cerglas bleus dévorent la laideur harassée.

Là, ces poutres éventrées me font pleuvoir au ventre les verglas neurotics de leur vermoulüre.

Pâle est la contrée de bouleaux, anémique est le sol, sanglante est la buée de mes genoux!

L'année est un glauque jour d'automne où les feuilles hypnosées ont des cancers exorbitants.

J'ai soif! Le mur m'est un scenic circulaire où les épinglettes de platine confectionnent à mon crâne la couronne de l'agonie.

Plafond aux pinces de condor! Aux crocs de coran, aux tenailles de dominique!

La morale aux prostates malades m'a crucifiée sur les lits du fakir! Oh! Et ce père abominable qui, dans la corbeille des mœurs, a taché le moka brun et doux!

Je suis une bête, un cornu buffle de cirque!

Qu'éclate le porto fermenté! Que la basilique aux cils noirs de cyclope s'écroule dans les démangeaisons de la peste!

Que la tuerie des annulaires flexibles et poudrés s'étende au monde entier! que la brûlure de mes flancs excédés suffoque le nain maigre comme la folle hydropique!

Je veux venger l'élance-pathie de mon clitoris! Les aiguilles du tourment clament aux étendues bâillonnées l'injustice du cadavre trépignant! Ah! Douleur!

Ingrates vermines! Que l'étole empaumée et transie des menstrues miaule dans une diarrhée de luxure! 
Le pôle me frappe, le flagellant nové me heurte au coin de ma cage, mais qu'importe! le délire de mes anus enfilés supplée à la malédiction des crachats teintés d'iode!

De mes cratères sublongiaux s'évadent des fibres volantes qui deviendront tourmenteurs des ingrats!

Le temps présent et l'amour sont fracassés dans un casse-noisettes d'acajou. La torpeur se civilise et répand son arôme poissonneuse en solution poudreuse.

Poudrure poudrette poudraline. C'est en ce magot de réticences abnéguées que l'ix des géomètres désigne sobrement les clous de mon écartèlement. Je veux mourir. Je veux mourir, ou je veux aspirer dans mes pompes l'huile d'érable que les gaules baptisées furelutent dans l'immersion! La frelature de la boucherie spermoaire déracinera la pulpe des fossettes ventrales! O tuer, égorger, noyer!

Annihiler le pape où flipotent les tarzans de la nuit!

Dans une aubépine aux cicatrices de sanctuaire, dépenailler les tendres lobes du cœur!

Exorciser la tempête aux matraques de verges! Exorciser le goéland qui dégonfle son pneu! Exorciser le diable qui n'est que la broussaille qu'humanise la mecque!

Planer dans la culture d'algues qui n'est qu'un cocuage, embrasser le serpent à sonnettes qui plongerait sa queve dans l'amphore athlétique! Ah! à quoi bon équilibrer des bulles de sarriette, mais le tréfonds de sarcophage ne réduira nul satyre à l'inertie des gelées!

la phygie est dressée, et l'écume grignote!

GAUVREAU, C., 1977 : OEuvres créatrices complètes, Montréal, Parti Pris, pp. 200-201.

C Succession Claude Gauvreau 


$$
\text { 的国 }
$$

